\title{
Design and Evaluation of a Layered Handheld 3D Display with Touch-Sensitive Front and Back
}

\author{
Patrick Bader, ${ }^{a b}$ Valentin Schwind, ${ }^{a b}$ Niels Henze, Stefan Schneegass, Nora Broy, ${ }^{b}$ Albrecht Schmidt $^{b}$ \\ ${ }^{a}$ Stuttgart Media University \\ Stuttgart, Germany \\ \{baderp, schwindv\}@hdm-stuttgart.de \\ ${ }^{b}$ VIS, University of Stuttgart \\ Stuttgart, Germany \\ \{firstname.lastname\}@vis.uni-stuttgart.de
}

\begin{abstract}
Touch screens became truly pervasive through the success of smartphones and tablet PCs. Several approaches to further improve the interaction with touch screens have been proposed. In this paper we combine and extend two of these trends. We present a mobile 3D screen that consists of a stack of displays and is touch sensitive on both display sides. This design makes the screen independent from the user's view angle. Using a touch-sensitive back enables back-of-device interaction to avoid the fat-finger problem. Combining backof-device interaction with a transparent display also avoids occlusion of the user's finger on the back through the device. Through a study we investigate how back and front touch improves interaction with 3D content and show how back-ofdevice interaction is improved if the user can actually see the finger on the back.
\end{abstract}

\section{ACM Classification Keywords}

H.5.2 [Information interfaces and presentation]: User Interfaces. - Graphical user interfaces.

\section{Author Keywords \\ 3D display, touch input, back-of-device interaction, mobile HCI \\ INTRODUCTION}

Over the last decade, touch screens have arguably become the most common way to interact with computing devices. Today, they are integrated in devices with a broad range of form factors. Through the success of smartphones and tablet PCs touch input became at least as common as the use of mouse and keyboard. In recent years, touch screens got thinner and their resolution increased. We currently see two further trends in mobile display technologies. On the output side, devices with 3D displays, such as the HTC Evo 3D, are emerging on the market which results in an additional degree of freedom for output [3] and in a better user experience [4]. On the input side, first devices do not only have touch screens but devices, such as the Sony Vita, also have a touch sensitive back.

Permission to make digital or hard copies of all or part of this work for personal or classroom use is granted without fee provided that copies are not made or distributed for profit or commercial advantage and that copies bear this notice and the full citation on the first page. Copyrights for components of this work owned by others than the author(s) must be honored. Abstracting with credit is permitted. To copy otherwise, or republish, to post on servers or to redistribute to lists, requires prior specific permission and/or a fee. Request permissions from Permissions@ acm.org.

NordiCHI '14, October 26 - 30 2014, Helsinki, Finland

Copyright is held by the owner/author(s). Publication rights licensed to ACM.

ACM 978-1-4503-2542-4/14/10 ...\$15.00.

http://dx.doi.org/10.1145/2639189.2639257

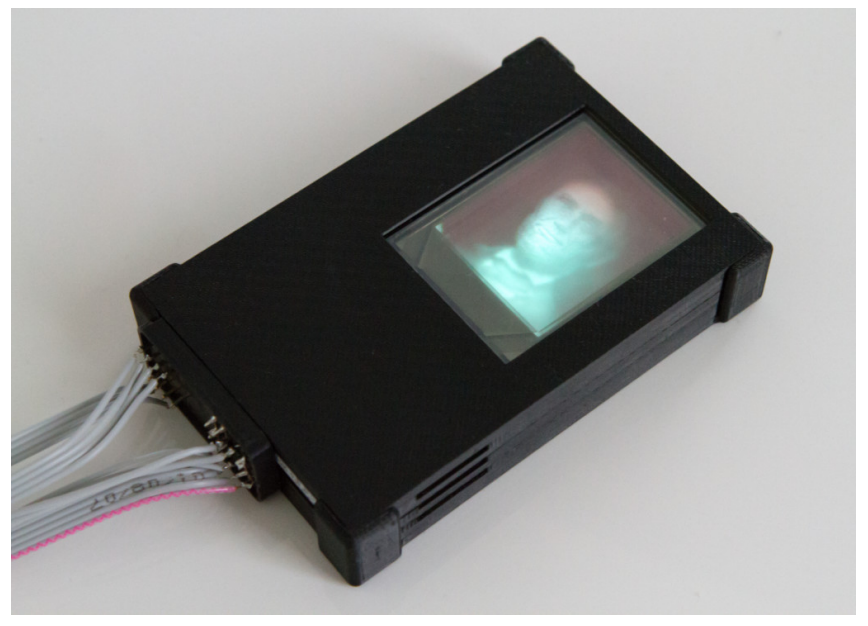

Figure 1: The developed handheld device with a layered 3D screen that enables touch input on both display sides.

With the fat-finger problem [7], traditional touch screens have a potentially inherent limitation. The part of the displays where the user aims at becomes occluded as soon as the user's finger approaches the screen. Devices with a touch-sensitive back enable to use both device sides for input. Sugimoto et al. first explore two sided input on mobile devices by using a pen on the front and the user's fingers on the back [9]. As shown by Wigdor et al. [11], back-of-device interaction can thereby solve the fat-finger problem as well. They render the user's hand that is interacting at the backside of the device overlayed on the front screen.

Current commercial devices and most research prototypes, however, only realize virtual back-of-device interaction. While previous work showed that even such a virtual backof-device interaction outperforms other pointing techniques [12], this technique suffers from the drawback that the fingers' positions on the backside need to be captured either externally or reconstructed using the touch positions. Thereby, they introduce what could be called the 'invisible finger problem'. While the user can permanently see a target, the fingers remain invisible as long as they are heading towards it. This drawback is tackled by Ohtani et al. by presenting a transparent touch-enabled display [6]. This display, however, is neither 3D nor in a form-factor of a mobile device.

Just as back-of-device interaction adds an additional degree of freedom for input, 3D displays add an additional degree for output. Autostereoscopic 3D displays enable to experience 
3D content without requiring additional devices such as shutter glasses. However, these devices require an specific viewing angle for the full 3D experience. In contrast, displays using a number of stacked display layers allow a wider viewing angle. Wetzstein et al. for instance, developed a tomographic technique for image synthesis on attenuation-based light field displays [10]. Another approach by Gareth Bell [2] shows the prototype of a multi-layered LCD screen with variable depth. As an additional benefit, such 3D displays allow higher resolutions than conventional displays with auto-stereoscopic parallax barriers since they do not need to separate the pixels for each eye.

Nevertheless, a major challenge [8], at least from a humancomputer interaction perspective, still is to explore usable interaction techniques for 3D screens. In particular, for screens with small form factors current interaction techniques do not work [1] and an optimal way of interacting needs to be explored.

In this paper we discuss how back-of-device interaction can improve the interaction with $3 \mathrm{D}$ content. We developed a 3D screen that consists of a stack of transparent displays (see Figure 1). Thereby, displaying 3D content becomes independent from the user's viewing position. As the displays are transparent the user can partially see-through them enabling to see the fingers through the display. Using a touch-sensitive front and back, we can utilize the full potential of back and frontof-device interaction.

The contributions of this paper are two-fold: First, we present a mobile 3D touch screen with three stacked displays. Second, we investigate how back and front touch improves interaction with 3D content through a user study and show how back-of-device interaction is improved if the user can actually see the finger on the back.

\section{PROTOTYPE}

We developed a prototype of a handheld version of a stacked 3D displays (cf., Figure 1) to investigate the invisible finger problem. As shown in Figure 2, the prototype is based on three display layers and two touch layers.

\section{Display}

We use uTOLED-20-G2 transparent OLED displays by 4D Systems with a screen diagonal of $2^{\prime \prime}$ and a resolution of 128 x 160 pixels (106 dpi). Compared to LC-panels no external backlight is required. Transparent OLEDs also enable additive color blending between multiple layers making unlit regions transparent. The display is powered by a graphics processor capable of drawing $2 \mathrm{~d}$ shapes and blitting images stored on a micro-SD card. This processor can either be programmed directly or controlled remotely via a serial connection.

Even though using more than three displays improves 3D perception, the contrast is reduced by each additional layer. This is due to each display having a maximum transparency of approximately $50 \%$. Using three displays, the content on the backmost display is still recognizable while $3 \mathrm{D}$ perception is preserved.

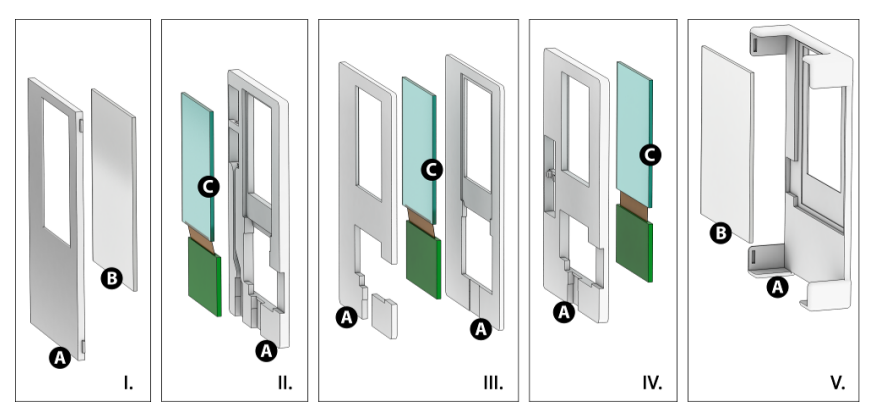

Figure 2: Explosion rendering of the stacked 3D-display device. The device consists of five independent Layers (grey background, numbered): front touch (1), front display (2), middle display (3), back display (4), back touch (5). Layers consist of case parts (A), touch sensors (B) and displays (C).

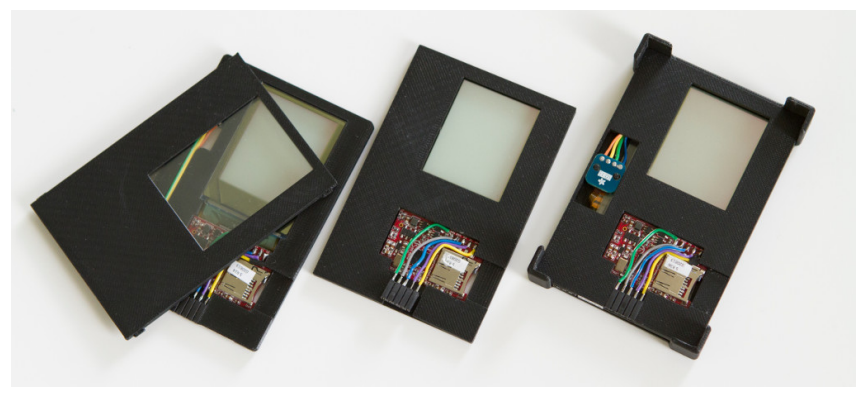

Figure 3: Disassembly of the display prototype showing the top layers with touch sensor and display (left), the middle display layer (middle) and the bottom layers with display and touch sensor (right).

\section{Touch Input}

Interaction is implemented by resistive touch sensors (Nintendo DSL digitizer) for both the front and the back side of the device. Thus interaction is limited to single-touch per sensor. Both sensors have an active diagonal of $3.2^{\prime \prime}$ and, thus, are larger than the screen.

\section{Display Assembly}

We set the spacing between each panel to $4.5 \mathrm{~mm}$ resulting in a total depth of $18.6 \mathrm{~mm}$ for the device including the touch sensors on both sides and the case. As shown in Figure 2, the front touch sensor lies directly on top of the front display so there is no gap between them. The back display panel and the back sensor are aligned likewise. In this way, we achieve a minimum error due to the parallax when displaying on the front panel and touching on the front sensor and displaying on the back panel and touching on the back sensor, respectively.

The enclosure of the prototype is 3D-printed and available as open-source ${ }^{1}$. The prototype consists of five independent layers. Each of these layers either contains a display or a touch-sensor (cf., Figure 3). The touch sensor layers were designed to restrict the touchable area to the size of the displays to guide users to the relevant touch area and to prevent unintended touches when holding the device.

\footnotetext{
${ }^{1}$ CAD-files and documentation are available at: https ://github. com/patrigg/Layered-3D-Display-Case
} 

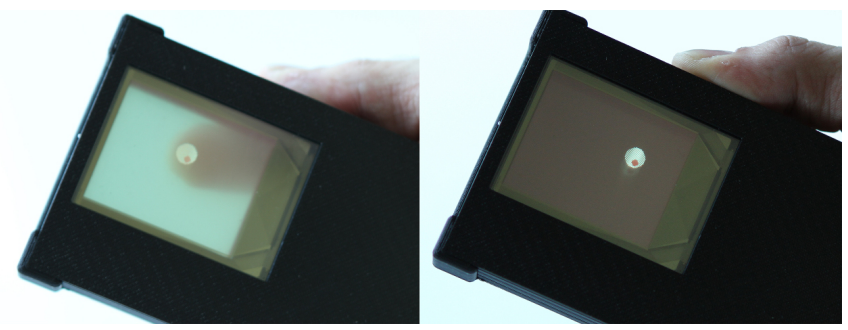

Figure 4: Typical task performed by a participant showing target (white circle), contact point (red circle) with back-touch in a see-through (left) and opaque (right) condition.

\section{Control}

The prototype is connected to a $\mathrm{PC}$ via a single microcontroller (mbed LPC1768) using the USB-serial interface for communication. Each display is connected to the microcontroller via RS232. Both touch sensors are connected using GPIO pins and directly measured by the microcontroller so no additional driver-IC is necessary. The microcontroller implements a simple multi-channel communication protocol and is responsible for dispatching messages between the PC and the corresponding display or sensor. Thus logically each display can be controlled directly from the application logic running on the PC.

\section{STUDY}

We conducted a user study to investigate how the users' touch performance is influenced by display transparency, the possibility to interact at both sides of the device, as well as the placement of content on different display layers. We used the previously described prototype throughout the whole study and asked participants to fulfill two parts including a number of target selection tasks. In the first part, targets were shown on one of the three display layers and participants selected the target from the front or the back. In the second part, participants performed the same task with targets only shown on the middle layer and the display either being transparent or opaque.

\section{Participants and Procedure}

We recruited 13 participants for the user study (3 female). Participants were mainly students with a technical background. Their average age was 27.54 years $(S D=3.69$, Median = 27). After the participants arrived at our lab, we introduced them the purpose of the study. Afterwards, the participant executed the first and the second part. For both parts the same target selection tasks had to be performed while holding the device like they usually hold a mobile phone. We presented white filled circles with a size of 21 pixels (i.e., $5.04 \mathrm{~mm}$ ) on the display the user should select. A task was completed when the user released their finger within the circle making it disappear. Note, that we allowed participants to drag the finger to the correct position or to retry the task until they succeeded. As soon as the user's finger touches the display, we provided visual feedback to the user by showing a blinking red circle (cf., Figure 4) with a size of 7 pixels (i.e., $1.68 \mathrm{~mm}$ ). We added a randomized delay ranging from 0.5 to 2 seconds between the tasks.

\begin{tabular}{lccccc}
\hline & \multicolumn{2}{c}{ Task Completion Time [ms] } & & \multicolumn{2}{c}{ Needed Trails } \\
\cline { 2 - 3 } \cline { 5 - 6 } Position & Front & Back & & Front & Back \\
\hline Front layer & $1527(448)$ & $1466(430)$ & & $1.51(0.31)$ & $1.52(0.38)$ \\
Middle layer & $1851(1170)$ & $1478(393)$ & & $1.77(0.74)$ & $1.42(0.25)$ \\
Back layer & $1953(1020)$ & $1494(544)$ & & $1.83(0.61)$ & $1.37(0.29)$ \\
\hline
\end{tabular}

Table 1: Mean (SD) of the task completion time and the needed trials from the first part of the user study.

\section{First Part}

The first part involves the independent variables number of display layers and touch side:

Display layers: As we expect that the display layer on which the target is displayed has an effect, we tested targets that appear on the front, middle, or back layer.

Touch side: For fulfilling the task the participants are instructed to react on the displayed targets by touching the device from the front or back,

The levels of the independent variables result in $3 * 2=6$ test conditions. We counterbalanced the test conditions with a balanced Latin square design. For each condition, the participants executed 20 target selection tasks. In total, each participant completed 120 tasks for the first part of the study. The display was always transparent during this task.

\section{Second Part}

In the second part, we fixed the output to the middle layer and varied the following independent variables:

Touch side: As in the first part, the participants should fulfill the task by touching the device from the front or back.

See-through: Moreover, we distinguish if the stacked displays are opaque or transparent. Thus, either all display layers are transparent or just the front two layers are transparent while the back one is opaque.

This study design results in $2 * 2=4$ conditions which we counterbalanced with balanced Latin square design. We presented 20 tasks for each condition, resulting in 80 tasks per participant for the second part in total. In addition, we collected subjective feedback by applying the AttrakDiff mini [5] questionnaire after each test condition. This standardized questionnaire assesses on the one hand usability and on the other hand user experience as well as the attractiveness of the user interface.

\section{Measures}

For both parts, we assessed three different measures. First, we measured the task completion time (TCT) - the time between the target is shown and the user selects it. Second, the distance between the initial touch down position and the target center. Third, the attempts necessary to fulfill the task.

\section{Results}

First Part

Table 1 shows the mean values and the standard deviation in each condition. We performed a repeated measures analysis of variance (ANOVA) that reveals a significant lower mean 


\begin{tabular}{|c|c|c|c|c|c|c|c|c|}
\hline \multirow[b]{2}{*}{ Position } & \multicolumn{2}{|c|}{ Initial Distance [pixels] } & \multicolumn{2}{|c|}{ Task Completion Time [ms] } & \multicolumn{2}{|c|}{ Needed Trials } & \multicolumn{2}{|c|}{ AttrakDiff } \\
\hline & Front & Back & Front & Back & Front & Back & Front & Back \\
\hline Transparent & $12.50(6.15)$ & $13.18(3.74)$ & $1152(234)$ & $1271(274)$ & $1.42(0.31)$ & $1.43(0.24)$ & $4.79,3.98,4.38$ & $4.96,4.00,4.50$ \\
\hline Opaque & $10.93(3.82)$ & $19.78(5.86)$ & $1321(261)$ & 1497 (428) & $1.47(0.26)$ & $1.96(0.82)$ & $4.83,4.15,4.35$ & $4.79,3.98,4,38$ \\
\hline
\end{tabular}

Table 2: Results from the second part of the user study. Mean (SD) of the initial distance, task completion time, and the needed trails as well as the pragmatic quality, hedonic quality, and attractivity from the AttrakDiff mini.

TCT for touching the back side than the front, $F(1,12)=$ 7.37, $p=.02, \eta^{2}=.38$. Also the mean amount of needed trials for the back touch condition is significant lower than with the front condition, $F(1,12)=6.20, p=.028, \eta^{2}=$ .34. There is no significant effect between the three display layers, $F(2,11)=.50, p=.62$.

\section{Second Part}

In the second part, we found that touch side, $F(1,12)=$ $19.22, p<.001, \eta^{2}=.62$, see-through, $F(1,12)=9.57$, $p=.01, \eta^{2}=.44$, as well as their interaction, $F(1,12)=$ $13.13, p=.003, \eta^{2}=.52$, have a statistically significant effect on the initial touch distance to the target center. The TCT is significantly lower with see-through compared to without see-through, $F(1,12)=10.97, p=.006, \eta^{2}=.48$. On the back, participants were more precise with see-through compared to without see-through. As depicted in Table 2 on the front, participants were more precise without seethrough compared to with see-through. We also found that see-through, $F(1,12)=3.02, p=.021, \eta^{2}=.20$, and its interaction with touch side, $F(1,12)=6.55, p=.025$, $\eta^{2}=.35$, have a significant effect on needed trials. On the back, participants needed less trials with see-through compared to without see-through. There is no significant effect between front and back touch, $F(1,12)=3.02, p=.108$, $\eta^{2}=.20$. The results from the AttrakDiff mini are shown in Table 2. We found no significant effects on the three dimensions (all $p>.05)$.

\section{CONCLUSION}

In this paper, we presented a handheld prototype of an autostereoscopic see-through 3D display. The device consists of multiple semi-transparent screen layers that form a volumetric display. The 3D output is independent from the user's view angle. Using a touch-sensitive back enables back-ofdevice interaction to avoid the fat-finger problem. Combining back-of-device interaction with a transparent display also avoids occlusion of the user's finger on the back through the device. The built device enabled us to conduct a controlled experiment to determine the effect of the screen layers, frontand back-touch as well as the transparency on touch performance. We found no significant effect of the screen layer on users' performance. Therefore, we assume that displaying content on different layers might have a negligible effect on the ability to select content through touch. Furthermore, the results reveal that a transparent display improves backof-device interaction compared to virtual transparency. Our work is an initial step towards interactive mobile volumetric 3D displays. We are particularly interested in the design space of layered handheld 3D displays. We assume that novel input techniques need to be explored.
Acknowledgements: This work was supported by the graduate program Digital Media of the Universities of Stuttgart and Tübingen, and the Stuttgart Media University (HdM).

\section{REFERENCES}

1. Alt, F., Schneegass, S., Auda, J., Rzayev, R., and Broy, $\mathrm{N}$. Using eye-tracking to support interaction with layered 3d interfaces on stereoscopic displays. In Proc. IUI (2014).

2. Bell, G. P. How deep is deep enough? In Cockpit and Future Displays for Defense and Security (2005).

3. Broy, N., Alt, F., Schneegass, S., Henze, N., and Schmidt, A. Perceiving layered information on $3 \mathrm{~d}$ displays using binocular disparity. In Proc. PerDis (2013).

4. Häkkilä, J., Posti, M., Koskenranta, O., and Ventä-Olkkonen, L. Design and evaluation of mobile phonebook application with stereoscopic $3 \mathrm{~d}$ user interface. In CHI-EA (2013).

5. Hassenzahl, M., and Monk, A. The inference of perceived usability from beauty. Human-Computer Interaction (2010).

6. Ohtani, T., Hashida, T., Kakehi, Y., and Naemura, T. Comparison of front touch and back touch while using transparent double-sided touch display. In Proc. SIGGRAPH (2011).

7. Siek, K. A., Rogers, Y., and Connelly, K. H. Fat finger worries: how older and younger users physically interact with pdas. In Proc. INTERACT (2005).

8. Steinicke, F., Benko, H., Krüger, A., Keefe, D., de la Riviére, J.-B., Anderson, K., Häkkilä, J., Arhippainen, L., and Pakanen, M. The 3rd dimension of chi (3dchi): touching and designing $3 \mathrm{~d}$ user interfaces. In CHI-EA (2012).

9. Sugimoto, M., and Hiroki, K. HybridTouch: an intuitive manipulation technique for PDAs using their front and rear surfaces. In Proc. MobileHCI (2006).

10. Wetzstein, G., Lanman, D., Heidrich, W., and Raskar, R. Layered 3D: tomographic image synthesis for attenuation-based light field and high dynamic range displays. In Proc. SIGGRAPH (2011).

11. Wigdor, D., Forlines, C., Baudisch, P., Barnwell, J., and Shen, C. Lucid touch: a see-through mobile device. In Proc. UIST (2007).

12. Wolf, K., and Henze, N. Comparing pointing techniques for grasping hands on tablets. In Proc. MobileHCI (2014). 